\title{
Bayly, Christopher A.: Remaking the Modern World 1900-2015. Global Connections and Comparisons (The Blackwell History of the World), 424 S., Wiley-Blackwell, Hoboken/NJ 2018.
}

\section{Angelika Epple}

Angenommen: 5. Januar 2021 / Online publiziert: 21. Januar 2021

(C) Der/die Autor(en) 2021

Als Christopher Bayly im April 2015 plötzlich verstarb, so schreibt der Reihenherausgeber R. I. Moore, habe er ein zwar nicht abgeschlossenes, aber eigentlich komplettes Buch hinterlassen (S. XI). Der Band „Remaking the Modern World 1900-2015“ konnte daher posthum in derselben Reihe wie das in der Zwischenzeit zum Klassiker avancierte Buch Baylys ,,The Birth of the Modern World. 1780-1914“ (2004) erscheinen. Wie stark die beiden Bände im Gespräch miteinander stehen, zeigt sich nicht nur durch die Anklänge der Haupttitel „Birth of“" und „Remaking the Modern World“ - „Remaking“ dabei in anderer Farbe hervorgehoben - sondern auch am identischen Untertitel: „Global Connections and Comparisons“.

2004 war der Untertitel noch programmatisch zu verstehen. Die Betonung der „Connections“" unterstrich Baylys Hauptanliegen zu zeigen, wie Verbindungen und Verflechtungen die Welt zwischen 1780 und 1914 enger zusammenrücken ließen. „Connections“ waren der Untersuchungsgegenstand. Die zunehmende Nähe und Verdichtung der Verbindungen führten, so der Autor, auch zu einer Angleichung der weltweiten Handlungsweisen. Die Welt wurde sich demnach ähnlicher. Der Begriff der „Comparisons“ wies hingegen nicht auf die Empirie, sondern auf die Methodik. Während in der Zwischenzeit die programmatische Intervention aus dem Jahr 2004 ihre Zähne verloren hat, da Globalgeschichte und die Analyse von Verflechtungen keine Besonderheit mehr darstellen, ist die Methodik der (welt-)regionalen Vergleiche ein Quell neuer, oft unverhoffter Erkenntnisse. Ein streitbares Narrativ, wie es der frühere Band geliefert hatte, lässt sich jedoch nicht erkennen.

Der aktuelle Band kann allerdings als Antwort auf drei thematische und theoretische Herausforderungen der Globalgeschichtsschreibung gelesen werden: Erstens die wachsende globale Ungleichheit, zweitens den Klimawandel und drittens die Kritik an der Globalgeschichte. Über Thomas Pikettys - laut Bayly zutreffender Diagnose der wachsenden Einkommensungleichheit hinausgehend, besteht Bayly daher erstens auf einer Erweiterung des Ungleichheitsbegriffs um Kriterien wie ,inhereted status“, ,ethnicity“ und „race and so-called tribe“ (S. 3). Nur dann könnten

\footnotetext{
A. Epple ( $\bowtie)$

Universität Bielefeld, Bielefeld, Deutschland

E-Mail: aepple@uni-bielefeld.de
} 
beispielsweise die Armut der schwarzen Bevölkerung oder geschlechtsspezifische Unterschiede erklärt werden. Der Umweltgeschichte widmet er zweitens ein eigenes Kapitel mit dem Titel „The Pressure of People“ (Kapitel 17). Vor allem ist das Buch jedoch eine Antwort auf die dritte Herausforderung: die gängige Kritik an der Globalgeschichte. Globalgeschichte lasse außer Acht, was sich alles nicht verbinde, was sich nicht bewege, sie verliere Akteure aus dem Blick und führe entweder durch die Hintertüre kapitalistische oder westliche liberale Ideen in die Geschichte ein, oder lasse nicht erkennen, inwiefern das Ganze (die Globalgeschichte) mehr darstelle als die Summe ihrer Teile (Einzelstudien).

Bayly reagiert auf die Vorwürfe, indem er aus der Methodik des Vergleichens neue Funken schlägt und auf einen ,moral imperative for historians“ (S. 5) abhebt. Dass große Teile der Welt von Krieg und Armut geprägt seien, führt er auf ambivalente Prozesse zurück: Er zeichnet nach, welche Ideen sich (nicht) durchsetzten, wer von Netzwerken ein- und wer ausgeschlossen wurde, welche Verbindungen (nicht) geknüpft wurden. Zwar sei der Wandel im 20. Jahrhundert durch viele Komponenten angestoßen worden - Kapital, Kontrolle über Arbeit, den modernen Staat, Imperialismus, Religion und Säkularisierung, Kommunikation - die entscheidende Prägung aber habe das Jahrhundert durch Kriege erfahren. Der Zeit der Massenvernichtung zwischen 1914 und 1945 (S. 12-117) folgten die Dekolonisierung und der Kalte Krieg bis 1990 (118-179); ihnen schlossen sich chronologisch die ,small wars of fragmentation“ an, die auch die Gegenwart prägten (S. 298-322).

In den Kapiteln 10-17 des Buches (S. 179-297) werden querliegende Themen wie zum Beispiel Kunst, Literatur und Unterhaltung, der Schatten des Empires in der modernen Welt oder das Verhältnis von Person und Gesellschaft behandelt. Hier löst sich die Chronologie zugunsten sich überlagernder Felder auf. Statt von einer Uniformierung, wie sie die Welt im langen 19. Jahrhundert prägte, liest man nun von heterogenen, vielschichtigen Entwicklungen, häufig von Wissenstransfer und regionalen Weiterentwicklungen. Die Kapitel sind unterschiedlich durchgearbeitet. Unter den Stichwörtern ,the human, demography, religion“ (S. 179) werden beispielsweise die Wissenschaftsgeschichte, die Geschichte der Anthropologie und anderer Wissenssysteme beschrieben und zu übergreifenden, teilweise gewagten Thesen verbunden: ,The general tendency throughout the century was the move from a diffusionist understanding of cultural change to one which emphasised the inherent creative capacity of different societies, a shift which again contributed to the global rise of nationalist and ethnic self-assertion" (S. 184).

Für beide Buchteile gilt, dass historische Zäsuren, Akteur_innen und Schwerpunkte überwiegend der europäischen Geschichte entnommen sind, auch wenn der dieser Anlage inhärente Eurozentrismus zugleich mit beeindruckender Gelehrsamkeit konterkariert wird: „Decentralising the World“ ist das Leitmotiv, das auch diesen Band durchzieht (S. 98). Das Buch ist überaus informativ, bindet alle Weltregionen ein, zeigt Gemeinsamkeiten wie Unterschiede und weitet den Blick, sodass auch die europäische Geschichte in neuem Licht erscheint, ist aber - wie bereits der erste Band - nicht dezentriert im Sinne der postcolonial studies. Europa erscheint nicht nur als versteckter ,,silent referent“ (Dipesh Chakrabarty), sondern meist als ,central referent". 
$\mathrm{Zu}$ gerne hätten wir gewusst, wie Christopher Bayly dieses überreiche Buch tatsächlich abgeschlossen hätte. Vermutlich wäre es deutungsfreudiger, stringenter und im besten Sinne streitbarer geworden.

Funding Open Access funding enabled and organized by Projekt DEAL.

Open Access Dieser Artikel wird unter der Creative Commons Namensnennung 4.0 International Lizenz veröffentlicht, welche die Nutzung, Vervielfältigung, Bearbeitung, Verbreitung und Wiedergabe in jeglichem Medium und Format erlaubt, sofern Sie den/die ursprünglichen Autor(en) und die Quelle ordnungsgemäß nennen, einen Link zur Creative Commons Lizenz beifügen und angeben, ob Änderungen vorgenommen wurden.

Die in diesem Artikel enthaltenen Bilder und sonstiges Drittmaterial unterliegen ebenfalls der genannten Creative Commons Lizenz, sofern sich aus der Abbildungslegende nichts anderes ergibt. Sofern das betreffende Material nicht unter der genannten Creative Commons Lizenz steht und die betreffende Handlung nicht nach gesetzlichen Vorschriften erlaubt ist, ist für die oben aufgeführten Weiterverwendungen des Materials die Einwilligung des jeweiligen Rechteinhabers einzuholen.

Weitere Details zur Lizenz entnehmen Sie bitte der Lizenzinformation auf http://creativecommons.org/ licenses/by/4.0/deed.de. 